\title{
Development of a rapid-scan fiber-integrated terahertz spectrometer
}

\author{
Hakan Keskin • Hakan Altan • Seydi Yavas • \\ Fatih Omer Ilday · Koray Eken • Asaf Behzat Sahin
}

Received: 30 April 2013 / Accepted: 1 October 2013 / Published online: 11 October 2013

C Springer Science+Business Media New York 2013

\begin{abstract}
Scientists in terahertz (THz) wave technologies have benefited from the recent developments in ultrafast laser technologies and RF technologies and applied these new gained techniques into characterizing a wide variety of phenomena. Undoubtedly, the most successful of these applications has been in the development of time-domain terahertz spectroscopic and imaging systems which has been utilized in the characterization of dielectrics and semiconductors. This pulsed technique has allowed users to characterize dynamical behavior inside materials under illumination with picosecond resolution. Typically pump/probe or similar dynamical measurements require the use of amplified pulses derived from free-space solid state lasers in the $\mu \mathrm{J}-\mathrm{mJ}$ range and since interferometric techniques are typically used in pulsed measurements the measurement time of a THz spectrum can last at least tens of minutes. Better systems can be realized based on fiber laser technologies. Here we discuss the advantages of a $\mathrm{THz}$ spectrometer driven by an ultrafast Ytterbium doped fiber laser whose repetition rate can be tuned rapidly allowing for rapid dynamical measurements. The efficient gain medium, robust operation and compact design of the system opens up the possibility of exploring rapid detection of various materials as well as studying dynamical behavior using the high brightness source.
\end{abstract}

Keywords THz $\cdot \mathrm{TDS} \cdot \mathrm{TRTS} \cdot$ Fiber laser $\cdot$ Rapid scan

\section{H. Keskin $(\bowtie) \cdot$ H. Altan}

Department of Physics, Middle East Technical University Cankaya, 06800 Ankara, Turkey

e-mail: keskin.hakan@metu.edu.tr

\section{S. Yavas · F. O. Ilday}

Department of Physics, Bilkent University Cankaya, 06800 Ankara, Turkey

K. Eken

FiberLAST ODTU Teknokent Galyum Blok Z-3, 06800 Ankara, Turkey

A. B. Sahin

Department of Electrical and Communication Engineering, Yildirim Beyazit University, Altindag, 06030 Ankara, Turkey 


\section{Introduction}

Pump/probe spectroscopy is a useful tool to investigate dynamic events on microscopic scales in metals and semiconductors. To date, many materials have been examined by visible and near-infrared lasers in pump/probe experiments (Baxter and Schmuttenmaer 2009). With the advent of time-domain techniques in the terahertz region such as THz-Time Domain Spectroscopy (THz-TDS) these methods have scaled to the far-infrared region with some calling femtosecond visible excite/THz probe measurements as one of the best tools to investigating carrier dynamics in nanoscale materials (Beard et al. 2002). The application of these methods into this region where sample architectures are dictated by empty gaps or vast areas of dielectric background amongst a rare presence of nanomaterial have driven system architectures which require special tools. Typically, these systems require high power laser sources in order to produce high level of carriers in these samples to change probe characteristics. When pump/probe systems are utilized with terahertz generation studies, it is common to derive the pulse for the generation and detection of $\mathrm{THz}$ radiation as well as excitation of the sample from the same laser source (Beard et al. 2000). Furthermore, generation of $\mathrm{THz}$ transients with broad spectral range is governed by the use of mode-locked lasers with pulse durations less than 200 fs duration (Beard et al. 2000). These lasers typically have pulse energies in the $\mathrm{nJ}$ range for repetition rates (rep-rate) in the tens of $\mathrm{MHz}$ range. Therefore amplified lasers within the $\mu \mathrm{J}-\mathrm{mJ}$ range are generally required to perform pump/probe with $\mathrm{THz}$ pulses as the probe in typical experiments (Baxter and Schmuttenmaer 2006). However, these lasers are bulky, expensive, and need external cooling systems based on chillers. Coupled with the THz-TDS system the overall set-up can be quite complex and require constant calibration and fine adjustment to achieve adequate results. In addition, two or three optical arms required according to scan type with $\mathrm{THz}$ probe technique which increases the complexity of experiment setup. Recently, much progress has been in the development of mode-locked sub-200 fs fiber lasers with average power necessary to obtain nJ level energies per pulse. These lasers are attractive due to their compact design; robust performance and overall stability as characterized by the observed pulse to pulse jitter which is far better than any solid-state mode-locked femtosecond laser source based on passive mode-locking techniques which use dynamical elements to achieve mode-locking (Ilday and Wise 2002). The one of advantage of these systems is that their inherent low jitter allows the use of pulses other than the fundamental pulse in the same pulse train to perform THz measurements (Hochrein et al. 2010). In this manner, all fiber designs for $\mathrm{THz}$ spectroscopy can be implemented that do not require freespace propagation and the use of mechanical delay stages (Wilk et al. 2011). However, these systems lack the energy per pulse requirements necessary for achieving pump/probe experimental designs incorporating THz probe techniques. By using a highly efficient laser gain medium such as ultrafast Ytterbium (Yb):doped fibers and thermal management techniques based on double-clad fiber architectures recent developments have driven these fiber laser system to energy levels per pulse on par with traditional solid-state amplified laser sources. Coupled with measurement techniques developed in all-fiber laser systems, these developments have allowed for the proposed study in this work, which is an amplified Yb:doped fiber laser driven THz-TDS system that can perform pump/probe measurements in a more compact and robust platform. In this study, repetition rate tuning, power, spectrum and pulse duration stability of the constructed Yb-doped fiber laser oscillator were measured. The system that we are developing will allow for rapid measurements of $\mathrm{THz}$ pulse profiled which will be essential for scanning the entire $\mathrm{THz}$ waveform through the excited sample, a technique commonly referred to as $2 \mathrm{D}$-scan excite/THz probe measurements. These techniques are discussed in the context of the developed fiber laser system throughout the paper. 


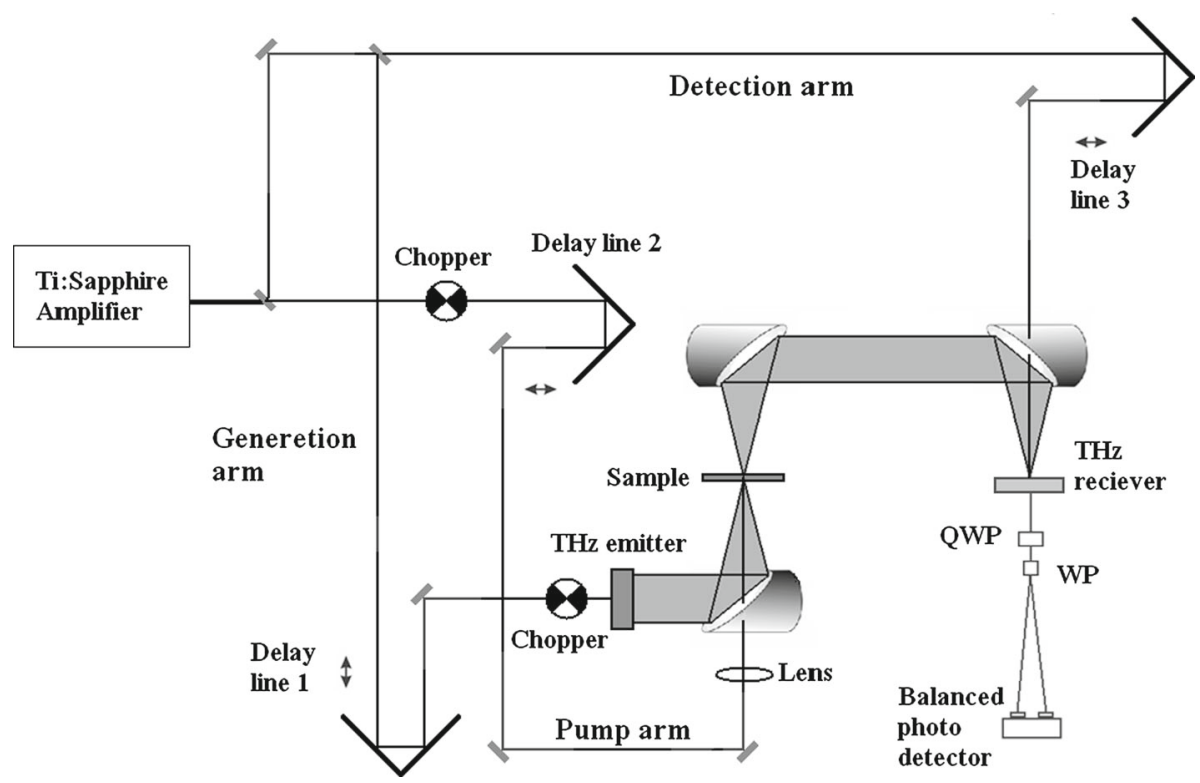

Fig. 1 Typical time resolved terahertz spectroscopy setup based on amplified Ti:A12O3 laser. QWP quarter wave plate. WP Wollaston prism

\section{Background}

For last two decades, time resolved terahertz spectroscopy (TRTS) has become an exceptional technique to understand the dynamic behaviors of nanomaterial, dielectrics and semiconductors (Ulbricht et al. 2011). In Fig. 1, a basic TRTS system setup is given with an Yb-doped fiber laser (YDFL) source. In this system, photoconductive antenna (PCA) is used for THz generation and electro-optic sampling is preferred as the detection method.

The pump/probe measurement technique can be implemented in reflectance or transmissive mode, in both cases it is important to be able to coincide both spatially and temporally the excite pulse and the THz probe pulse. The THz region employed in these measurements is typically sensitive to a specific energy region of a few to about $100 \mathrm{meV}$. In order to accurately characterize the material properties under photo-excitation, data needs to be obtained from this entire region. Traditionally, however, techniques that have been developed and are the easiest to implement that are frequency insensitive. These techniques are based on scanning either the probe or pump beams while the other one is fixed. These as well as frequency dependent techniques, are basically defined as 1D and 2D scan, respectively (Beard et al. 2000). In 1D scan, one delay line is scanned while holding the other arm fixed. A 1D pump scan provides useful information about the average $\mathrm{THz}$ absorption by the sample. To perform $2 \mathrm{D}$ scan, a THz difference scan at a series of pump-probe delay times is collected. These two techniques and their sub-classes are shown in Table 1.

As was mentioned previously $\mu \mathrm{J}-\mathrm{mJ}$ pulses are necessary to excite enough carriers in materials to see a response in the transmission or reflection of the probe beam. Yb-doped fiber laser amplified sources are typically required for this reason especially for thin samples because of low level of response. This reveals another condition for laser source. A stable laser system that has low jitter is a necessity. In our work, a fiber laser whose gain medium is 
Table 1 Scan types

\begin{tabular}{llllll}
\hline & \multicolumn{2}{l}{ 1D Scan } & & \multicolumn{2}{l}{ 2D Scan } \\
\cline { 2 - 3 } \cline { 5 - 6 } & Probe scan & Pump scan & & 1st Method & 2nd Method \\
\hline Pump & Fixed & Scanned & & Fixed & Scanned (synchronously) \\
Detection & Scanned & Fixed & & Fixed & Scanned (synchronously) \\
Generation & Fixed & Fixed & & Scanned (in reverse) & Fixed \\
\hline
\end{tabular}

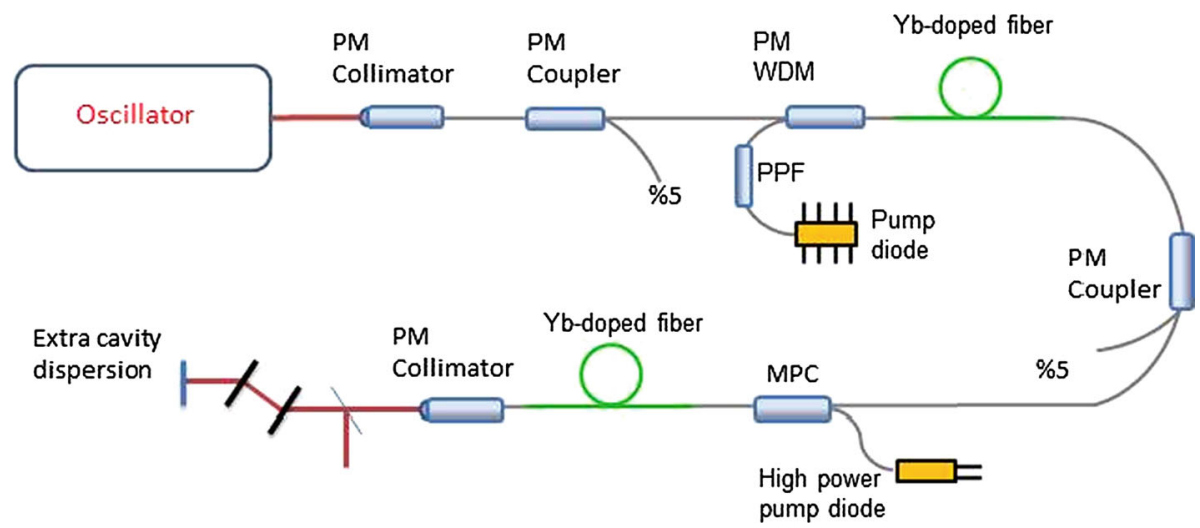

Fig. 2 Yb-doped fiber laser system. $P M$ Polarizing maintaining. MPC Multimode pump-signal combiner

efficient Yb-doped fiber for wavelength about 1,030 nm is used to obtain an amplified laser which has stable operation and low jitter. It possible to achieve $\mu \mathrm{J}-\mathrm{mJ}$ pulses with two stages of amplification with Yb-doped fiber (Mukhopadhyay et al. 2009). Our amplification design is shown in Fig. 2.

Polarization maintaining components should be used to create linearly polarized beams from amplification output. Output of oscillator is coupled into fiber via coupler and seed beam coming from oscillator is amplified in Yb-doped fiber. Pump diodes which emits light near $975 \mathrm{~nm}$ is used to pump the gain medium. After the first stage, typically the second stage of amplification consists of Multi-Port Combiner (MPC) and double clad (DC) Yb-doped fiber in which second clad of DC fiber enables to pump more power than one watt into core of gain medium in other words core of Yb-fiber. With this method, one can couple more than one high power pump diode in to the system via the MPC. Using these techniques, over $10 \mathrm{~W}$ of average power can be achieved with just two amplifier stages producing $\mathrm{nJ}$ level pulses (Mukhopadhyay et al. 2009). However, using additional amplification stages with similar design it has been shown to be possible to achieve sub- $\mu \mathrm{J}-\mu \mathrm{J}$ level pulses (Kalaycioglu et al. 2011). Finally, positively chirped pulses are compressed by using passive grating compressor schemes. Typical parameters at output are sub-200 fs pulses with over$100 \mathrm{~nJ}$ energy at a repetition rate of $50 \mathrm{MHz}$. The high rep-rate is beneficial to handle with non-linear effects in fibers due to high average power. Cooling is also important in overall stability and performance where a peltier device or passive cooling system can be used to stabilize temperature of the pump diodes. Overall, the level of energy per pulse and the stability of the system allow it to be incorporated into parametric generation schemes to obtain a broad range of excitation wavelengths. Recently, techniques based on this technology have 
evolved rapidly and Yb:doped mode-locked fiber laser systems are being combined with non-linear optical techniques in OPA configuration to produce versatile systems (Tzeng et al. 2009).

\section{Fast scan THz detection methods}

In conventional THz-TDS, THz profile of pulses is measure by scanning time delay between generation and detection arms in THz spectroscopy systems (Grischkowsky et al. 1990). This method can take several tens of minutes to complete depending on the scan length. Therefore, it is highly affected by fluctuations of source and environments. New techniques have been developed to shorten the measurement times. One of them is the asynchronous optical sampling method (Yasui et al. 2005). In this method, two lasers are used. Their repetition rates are locked with an offset $\left(\Delta \mathrm{f}=f_{\text {rep } 1}-f_{\text {rep } 2}\right)$. Using a piezo or similar to tune the one lasers cavity length the $\mathrm{THz}$ profile is measured instead of scanning the mechanical delay stage. One notable disadvantage is that, this kind of system requires the use of two mode-lock fs lasers which increases overall complexity and price of the setup. Moreover, complex systems based on RF techniques to control frequency stability are needed to stabilize the repetition rates of the lasers that are used. These investigations propelled researchers to develop a new technique called Optical Sampling by laser Cavity Tuning (OSCAT) (Hochrein et al. 2010). In the OSCAT technique, pulses that originate not from the same but from separated optical pulses are superimposed by scanning the repetition rate of a fiber laser. This type of measurement technique has been applied in $\mathrm{THz}$ spectrometers driven by Er-doped mode-locked fiber lasers (Wilk et al. 2011). Time delay between these two pulses can be calculated as:

$$
\Delta \tau=i\left[\frac{1}{f_{\text {ref }}}-\frac{1}{\left(f_{\text {ref }}+\Delta f\right)}\right]
$$

where $f_{\text {rep }}$ is repetition rate, $\Delta \mathrm{f}$ is change in repetition rate, $\Delta \tau$ is time between pulses, $\mathrm{i}$ is pulse index. To achieve desired time delay, length difference of arms is calculated as:

$$
l_{\text {fiber }}=\frac{\Delta \tau c_{0}\left(f_{r e f}+\Delta f\right)}{\Delta f n}
$$

where $l_{\text {fiber }}$ is necessary fiber length in one arm, $c_{o}$ is speed of light, $\mathrm{n}$ is fiber refractive index (Wilk et al. 2011).

While the Er:doped mode-locked fiber lasers which have used this technique can be implemented in an all fiber design, the use of the center wavelength emission near $1,030 \mathrm{~nm}$ as with the case of the laser being designed (see Figs. 2, 3) for this study requires the use of free-space optical elements. Nonetheless, the expected overall stability and high energy per pulse benefit makes it desirable to develop a THz-system based on the OSCAT technique using $\mathrm{Yb}$ as the gain medium of the driving laser. For such a system, the expected design parameters are displayed in Table 2.

Using the parameters listed in Table 2, the data acquisition for a $\mathrm{THz}$ waveform with at least 100 ps length is expected to be less than 1 min (Wilk et al. 2011).

TRTS system driven with the Yb:doped amplified laser system presented here is shown in Fig. 4. Pump arm is driven from the detection arm. THz is generated via PCA and $\langle 110\rangle-$ oriented GaP crystal is used in optical sampling. 


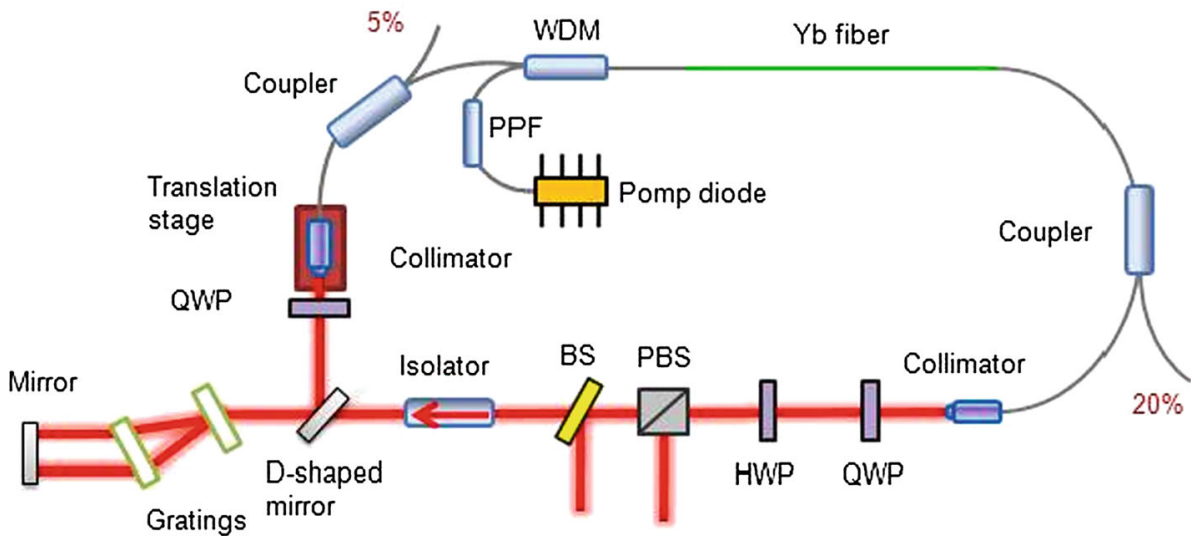

Fig. 3 Schematic representation of oscillator. $P P F$ Pump protection filter. WDM Wavelength division multiplexer. $Q W P$ Quarter wave plate. $H W P$ Halve wave plate. $P B S$ Polarizing beam splitter

Table 2 Yb:doped fiber laser scan parameters

\begin{tabular}{ll}
\hline & Yb:doped fiber laser \\
\hline Rep Rate $\left(f_{\text {rep }}\right)(\mathrm{MHz})$ & 50 \\
$\Delta \mathrm{f}(\mathrm{kHz})$ & 50 \\
$\Delta \tau(\mathrm{ps})$ & 100 \\
$n_{\text {fiber }}$ & 1,5 \\
Step size $(\mathrm{ps})$ & 0,1 \\
Stage movement inside laser $($ air $)(\mathrm{mm})$ & 6 \\
Pulse index (i) & 5,005 \\
$l_{\text {fiber }}$ (required fiber length) $(\mathrm{m})$ & 20,02 \\
\hline
\end{tabular}

By changing the generation time of the $\mathrm{THz}$ pulse using this OSCAT method implemented in a TRTS design as shown in Fig. 4 one can achieve the measurements as required by the $2 \mathrm{D}$ pump/probe scan method for either fixed pump pulse arrival derived from the detection pulse arm of the system (1st method in Table 1) or synchronous scanning of detection and pump arms for fixed generation (2nd method in Table 2). In both cases the scanning is being done with the OSCAT technique as described above, the mechanical stages allow for adjustment of $\mathrm{t}$ between the generation and pump pulse. These methods as outlined will allow for the rapid characterization of the dynamic events as observed for the sample over the full bandwidth of the $\mathrm{THz}$ pulse in a more elegant design.

\section{Measurements}

The Yb-doped fiber oscillator as shown in Fig. 3 produces $30 \mathrm{~mW}$ power output. Central wavelength of oscillator is about $1,040 \mathrm{~nm} 100 \mathrm{fs}$ pulses were derived from $\% 5$ output port. To implement the system in scheme as shown in Fig. 4, the stability of the oscillator was examined over the entire THz scan length by scanning the collimator over the range of $6 \mathrm{~mm}$. The measured repetition rate and power stability is plotted in Fig. 5. 


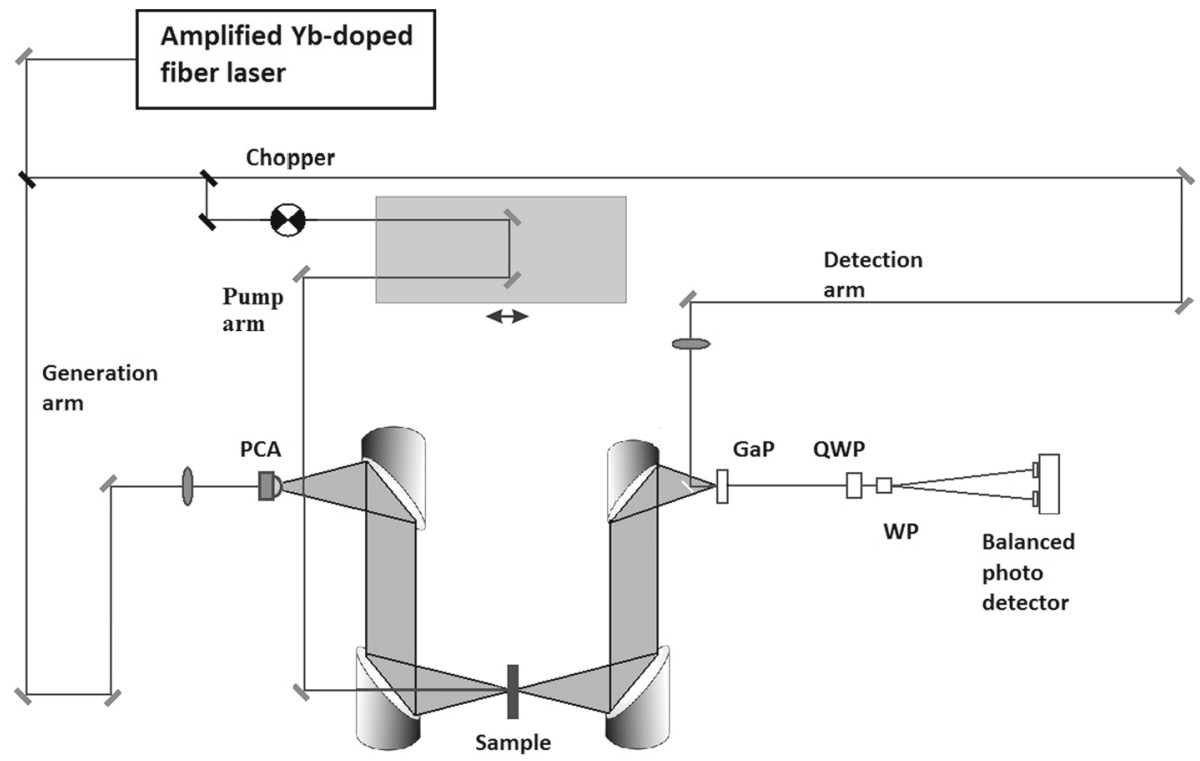

Fig. 4 TRTS system driven by Yb-doped fiber laser. Generation is scanned using the OSCAT technique while the detection arm and pump arm is held

(a)

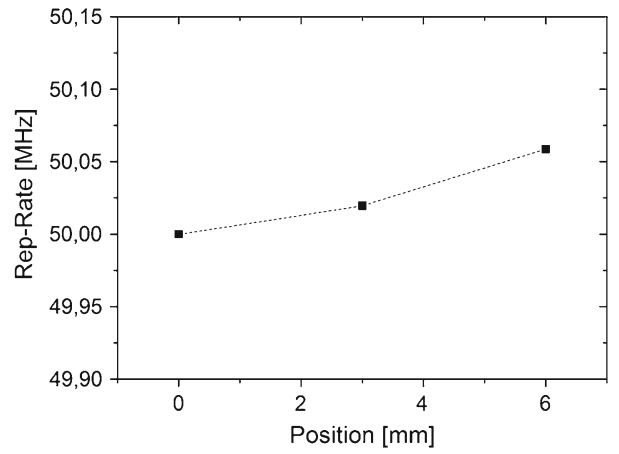

(b)

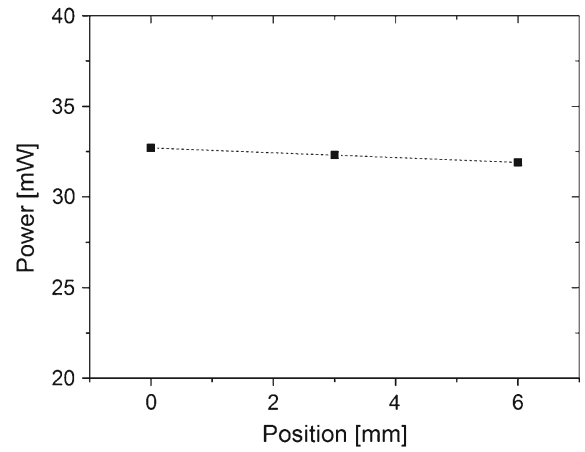

Fig. 5 a Repetition rate change; b Power fluctuation as oscillator cavity is scanned

At the same time in order to observe stability of laser oscillator spectra were recorded during the scan interval. The obtained spectra show that the output has a center wavelength of about 1,040 nm with a FWHM of $65 \mathrm{~nm}$ as shown in Fig 6a. Spectrum was observed to be stable with changing cavity length. Pulse durations for scan points are also measured as we scan $6 \mathrm{~mm}$ of cavity length. Autocorration (AC) signal of scanned pulses is show in Fig 6b. Intensity of oscillator output is normalized to 1 . Although there is some change at third position, the overall stability of the system is quite good with respect to expected measurements in the THz-TDS system. The pulse width change is below $1.01 \%$ and measured pulse durations of first, second and third positions are $965.05,969.47$ and 974.78 fs respectively assuming pulse shape is Gaussian. This pulse will seed the amplifier stages and subsequently be compressed before being used in the $\mathrm{THz}$ generation and detection arms as shown in Fig. 2. 

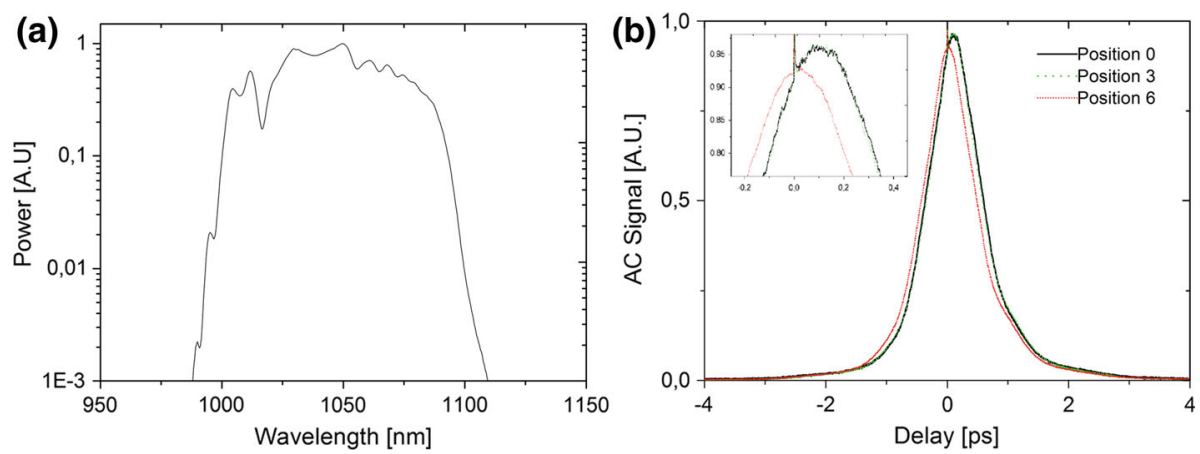

Fig. 6 a Spectrum output of oscillator; $\mathbf{b}$ pulse profiles obtained during the scan length

\section{Conclusions}

The development of a novel TRTS system driven by an Yb-doped fiber laser amplified laser is discussed specifically for excite/THz probe measurements. The system can scan the $\mathrm{THz}$ waveform without the use of any external delay lines, thereby shortening the data acquisition times throughout the measurements. The data acquisition is based on the OSCAT method (Hochrein et al. 2010) where the laser cavity repetition rate is tuned over the entire $\mathrm{THz}$ waveform. The oscillator for the amplified Yb-doped fiber laser system was constructed with a repetition rate of $50 \mathrm{MHz}$. In this study, repetition rate tuning, power, spectrum and pulse duration stability of the constructed oscillator were measured. After adding an additional amplifier to the pre-amplified Yb-doped fiber laser, it will be possible to perform fast scan TRTS measurements. To scan a length of $100 \mathrm{ps}$ the cavity needs to be tuned over $+/-25 \mathrm{kHz}$, for which the laser system exhibited little or no difference in output pulse duration, power and spectrum characteristics which is a testament to the stability of these lasers compared to other solid-state mode-locked lasers. Using this technique, scan times of obtaining one $\mathrm{THz}$ waveform for TRTS measurements is less than $1 \mathrm{~min}$. By using this characteristic, coupled with sub- $\mu \mathrm{J}-\mu \mathrm{J}$ output energy per pulse, the proposed fast scanning is discussed in the context of $2 \mathrm{D}$ excite/THz probe measurements, where it is shown that such a scan method is not only useful but essential for obtaining frequency resolved dynamical measurements of a variety of samples.

Acknowledgments This work is partially supported by The Scientific and Technical Research Council of Turkey under Grant \# 111T748.

\section{References}

Baxter, J.B., Schmuttenmaer, C.A.: Conductivity of $\mathrm{ZnO}$ nanowires, nanoparticles, and thin films using timeresolved terahertz spectroscopy. J. Phys. Chem. B 110, 25229-25239 (2006)

Baxter, J.B., Schmuttenmaer, C.A.: Carrier dynamics in bulk ZnO. II. Transient photoconductivity measured by time-resolved terahertz spectroscopy. Phys. Rev. B 80, 235206-1-235206-10 (2009)

Beard, M.C., Turner, G.M., Schmuttenmaer, C.A.: Transient photoconductivity in GaAs as measured by timeresolved terahertz spectroscopy. Phys. Rev. B 62, 15764-15777 (2000)

Beard, M.C., Turner, G.M., Schmuttenmaer, C.A.: Size-dependent photoconductivity in CdSe nanoparticles as measured by time-resolved terahertz spectroscopy. Nano Lett. 2(9), 983-987 (2002)

Grischkowsky, D., Keiding, S., Vanexter, M., Fattinger, C.: Far-infrared time-domain spectroscopy with terahertz beams of dielectrics and semiconductors. J. Opt. Soc. Am. B 7(10), 2006-2015 (1990) 
Hochrein, T., Wilk, R., Mei, M., Holzwarth, R., Krumbholz, N., Koch, M.: Optical sampling by laser cavity tuning. Opt. Express 18, 1613-1617 (2010)

Ilday, F.O., Wise, F.W.: High-energy femtosecond stretched-pulse fiber laser with a nonlinear optical loop mirror. Opt. Lett. 27(17), 1531-1533 (2002)

Kalaycioglu, H., Eken, K., Ilday, F.O.: Fiber amplification of pulse bursts up to $20 \mu \mathrm{J}$ pulse energy at $1 \mathrm{kHz}$ repetition rate. Opt. Lett. 36, 3383-3385 (2011)

Mukhopadhyay, P.K., Ozgoren, K., Budunoglu, I.L., Ilday, F.O.: All-fiber low-noise high-power femtosecond Yb-fiber amplifier system seeded by an all-normal dispersion fiber oscillator. IEEE J. Sel. Top. Quantum 15, 145-152 (2009)

Tzeng, Y.W., Lin, Y.Y., Huang, C.H., Liu, J.M., Chui, H.C., Liu, H.L., Stone, J.M., Knight, J.C., Chu, S.W.: Broadband tunable optical parametric amplifi- cation from a single $50 \mathrm{MHz}$ ultrafast fiber laser. Opt. Express 17(9), 7304-7309 (2009)

Ulbricht, R., Hendry, E., Shan, J., Heinz, T.F., Bonn, M.: Carrier dynamics in semiconductors studied with time-resolved terahertz spectroscopy. Rev. Mod. Phys. 83, 543-586 (2011)

Wilk, R., Hochrein, T., Koch, M., Mei, M., Holzwarth, R.: Terahertz spectrometer operation by laser repetition frequency tuning. J. Opt. Soc. Am. B 28(4), 592-595 (2011)

Yasui, T., Saneyoshi, E., Araki, T.: Asynchronous optical sampling terahertz time-domain spectroscopy for ultrahigh spectral resolution and rapid data acquisition. Appl. Phys. Lett. 87, 061101-1-061101-3 (2005) 